\title{
"Step" versus "Phase" in the clinical development of biosimilars: the correct noun holds the key to success
}

\author{
Reginald Ewesuedo \\ Pfizer Inc., Cambridge, MA, USA
}

Correspondence: Reginald Ewesuedo PO Box 40I16, San Antonio,

TX 78229, USA

$\mathrm{Tel}+\mid 210625$ | I7|

Email ejiagu@netscape.net
This article was published in the following Dove Press journal:

Biosimilars

22 June 2016

Number of times this article has been viewed

\begin{abstract}
The availability of biosimilars (also known as follow-on biologic or subsequent entry biologic, etc) is expected to improve access to health care and to provide more affordable alternatives to the reference products. However, despite this promise, based on publicly available information from clinical trial databases, there is apparent global disparity in participation (more so in oncology) and interest in the clinical development of proposed biosimilars. The disparity seems to be partly due to lack of access to biologics, as well as expectations of the development process and/or study objectives. There is inherent misalignment between the characterizations of the clinical studies, in the public domain, with regulatory guidance (eg, the use of "Phase" to describe a comparative study). There is also apparent non-appreciation of potential research opportunities afforded by the biosimilar development paradigm. It is imperative that key stakeholders required for the development of this class of drugs are adequately engaged and that they fully understand the development paradigm, if the promises of biosimilars are to be realized globally. Keywords: p-biosims, follow-on biologic, biologics, regulatory guidelines, clinical studies
\end{abstract}

\section{Expectations and opportunities with biosimilars development}

A biosimilar drug (also known as follow-on biologic or subsequent entry biologic in different regions) is not an exact copy of the innovator biologic. Nevertheless, it has similar structure, function, mechanism of action, and pharmacokinetic, pharmacodynamic, and risk-benefit profiles. Biosimilars are expected to improve access to health care and to provide more affordable alternatives to the reference products. Despite the promise of biosimilars, based on publicly available information (eg, Clinicaltrials.gov and TrialTrove), compared to new molecular entities, there is emerging global disparity, more so in oncology, in participation and interest in the clinical development of proposed biosimilars ( $\mathrm{p}$-biosims) by key stakeholders. The disparity seems to be partly due to lack of access to biologics, as well as gaps in knowledge and expectations of the development process that likely stems from misaligned description of the clinical studies undertaken in a typical clinical development program. There is also apparent non-appreciation of potential research opportunities afforded by the biosimilar development paradigm.

The development of a p-biosim is characterized by a sequence of research activities that are not typical for the development of a new molecular entity (NME). Unique to the development paradigm is that the clinical research activities are predicated on the need to resolve residual uncertainties due to (potential) concern over one or more attributes of the p-biosim. Inherent in the clinical development paradigm is the need 
to conduct studies that convincingly demonstrate similarity between the reference product and p-biosim, with the use of sensitive end point(s) that can discern the lack of similarities. This distinct difference between the clinical development of a NME and a p-biosim is apparently underappreciated by key stakeholders, and the paucity of understanding is susceptible to exacerbation by commonly used terminologies describing the clinical studies. The terminologies do not adequately characterize the "steps or stage(s) of development", and the scientific underpinnings that are required. By regulatory demands, the clinical development components of a p-biosim are much fewer compared to what would be typical for a NME. There are fewer clinical trials expected. These may include pharmacokinetics (PK), PK-pharmacodynamics (PK-PD), efficacy (one or more based on the reference product), safety, and immunogenicity studies. All or a subset of these studies combined are expected to contribute to the "totality of evidence" needed to demonstrate biosimilarity between the reference product and a p-biosim. The overarching goal in the development of a p-biosim is "to demonstrate biosimilarity between the proposed product (p-biosim) and a referenced/licensed product (innovator biologic)". To this end, there are guidelines from major regulatory agencies on the recommendations and requirements for what level of scientific evidence should support the development of a potential biosimilar. ${ }^{1-3}$

Although the regulatory guidelines are congruent and employ very similar and sometimes identical terminologies, the emerging literature and publicly available information describing the clinical development of biosimilars seem to be plagued by subtle inadvertent contradictions and/or misinterpretations of the "spirit" of the regulatory guidelines, eg, the use of "Phase" to characterize clinical trials and therefore the expectations by key stakeholders for routine elaborate clinical trials, supporting documents and processes utilized for the clinical trials are divergent. These publications and current practices present a set of challenges for stakeholders such as investigators, nurses, pharmacists, and patients who are required partners in the development process.

Before highlighting some of the contradictions, it is worth reviewing briefly the traditional terminologies and our common understanding of them when applied to the development of a NME. Conventional use of the term "Phase" in clinical development is intended as a "noun", and it invokes expectations of a set of clinical research objectives and end points based on the "Phase" of clinical development referenced as follows:
1. A Phase I study demonstrates if it is safe to administer a NME to humans. The primary objectives are typically "dose finding", which is the description of PK characteristics and safety evaluation after administration of single or multiple doses. Several Phase I studies including ten or more healthy volunteers and/or patients with durations that could last up to 2 years, depending on the disease of interest, are typical.

2. A Phase II study involves more clinical evaluation of the drug with the main objective of discerning activity and better characterization of the safety profile. The typical study could be single arm or randomized controlled trial and could involve up to 100 or more patients and last up to 3 years depending on the disease of interest

3. A Phase III study is characterized by the main objective to demonstrate a new treatment is better than an available treatment option. Study end points may be clinical benefit end points that are predictive or indicative of improvement in survival. These studies are typically randomized trials involving several hundreds of patients and could last several years, depending on the disease of interest.

The abovementioned characterization of the Phases of clinical trials implies the gradual unraveling of the benefit-risk profile of a NME. However, contrary to the objectives and end points of the various Phases of a conventional clinical trial, the prevailing terminologies characterizing the clinical development of p-biosims are not aligned with similar expectations, resulting in an apparent disconnect and a paucity of understanding and/or interest in potential benefits of the clinical research opportunities.

In order to create a gradual and meaningful assimilation of the clinical development paradigm of biosimilars, it is necessary to clarify and reposition the terminologies applied to the clinical development of biosimilars so as to be aligned with the regulatory guidelines, as well as better educate key stakeholders required for the development opportunity.

The initial "Step"/clinical study for most potential biosimilars is with a single dose. The main objective is the demonstration of PK similarity with the reference product through confirmation of bioequivalence. The dose for the study is not required to be a therapeutic dose. Repeated dosing or dose escalation is not a requirement for these studies. The demonstration of PK similarity at this initial step of clinical development is critical for continued development. This study is typically performed in a population that is the most homogeneous and/or most sensitive to discern similarity 
between the reference product and p-biosim. Despite these obvious demands, the literature characterizing most of these studies is incorrectly titled as "Phase I study".

To promote better understanding of the clinical development activity, it is reasonable to suggest that this study should only be referred to as Comparative Clinical PK study. Such a description conveys to the reader this is a PK study that is "not" akin to what is typically expected for a Phase I study, instead there is a known PK profile being elucidated for comparison in the study. Furthermore, it does not entail dose finding and/or selection; there is no need for a subset/ exploratory analysis of the data and no expectation of a clinical benefit signal which may drive the continued development of the p-biosim. Safety is usually not a primary objective at this stage of development of the p-biosim unless there are residual concerns emanating from preclinical data which are highly suggestive of the potential for disparity in safety profile between the reference product and p-biosim. The next study, if not an efficacy study, would typically evaluate the PD property(ies) of the p-biosim. Such a study when referred to as "Comparative Clinical Pharmacodynamics Study" should characterize a step in the development scheme, where PD can be integrated into the process. Finally, the "efficacy" study should be referred to as the "Comparative Clinical Efficacy Study". This study focuses mainly on two major objectives: primarily to demonstrate similarity with a clinically relevant and "sensitive" endpoint and secondarily safety and immunogenicity. This study is intended to demonstrate similarity using a typical clinical activity and/or end point for both the p-biosim and reference product. Supportive trials should be titled as "Supportive Clinical Comparability Study". This title indicates that the p-biosim is undergoing or has undergone other steps of development and demonstrated similarity to the reference product.

At no point in clinical development, should there be an expectation for subset analysis or characterization of an emerging benefit-risk profile for the reference drug or p-biosim. The nomenclatures proposed earlier should help to better characterize, and make it easier and less confusing to understand the studies undertaken for the clinical development of p-biosims.

The conduct of biosimilar studies presents a very unique opportunity for the scientific community to address questions including "interchangeability", lingering questions on mechanism(s) of action, previously unexplored activity, determinants of safety attributes, etc after regulatory approval of a biologic. Thus, the clinical development of a p-biosim presents a plethora of possibilities to address questions in the context of exploratory or confirmatory research using more recent knowledge and advanced technologies. New treatment paradigms could be explored especially in off-label or potential new indications.

Another terminology worth exploring is "extrapolation". Extrapolation is a topic of continued discussion in the development of a p-biosim. Simply defined, extrapolation infers drawing a conclusion based on known data, making an educated guess or prediction based on facts and observations about a present or known situation. Commentaries on extrapolation and interchangeability seem to be mushrooming into a call for more clinical trials. Ongoing review of the literature on extrapolation suggests an apparent gradual drift from the goals/intent of the development paradigm for biosimilars into a discussion on the need for extra studies to demonstrate similarity in clinical efficacy and safety in every indication for which the reference product is approved. While such positions merit close attention, it would seem that if the main basic pharmacological attributes of any two products are similar or superimposable - including, eg, receptor affinity and binding - the clinical outcome expected from perturbation of the receptor will be similar since dysregulation or disruption of downstream receptor signaling effects should be no different.

By definition, a biosimilar is not expected to be identical to the reference product. There are bound to be minor differences in quality attributes; however, the basic pharmacologic properties including target binding and PK are already known or demonstrated to be similar. Thus, the clinical activity is extremely unlikely to be significantly different from the reference product when used for the treatment of currently approved indication for the innovator.

Furthermore, assuming a hypothetical situation in which both a reference product and the proposed p-biosim are being evaluated at the preclinical Phase of an initial development program, it is unlikely that any known minor differences in quality attributes may warrant the development choice of one over the other. Either one will be considered acceptable for development for the target and/or indication(s). Therefore, a similar perspective can and should be applied retrospectively, especially where a clinical study has demonstrated high similarity in PK profile and/or PK-PD relationships. The "need" for more clinical studies should "not" become the gold standard. The argument can be made that comparative clinical efficacy studies should only be "supportive" and "not" a requirement in extrapolation decisions. Clinical efficacy studies are more likely to be less sensitive in discerning similarity between a p-biosim and reference product when 
compared to data from other studies. A clinical efficacy study is intended to address residual uncertainty.

Nevertherless, it is extremely unlikely that a p-biosim which demonstrated similarity in exposure and activity in one or more clinical settings/studies will depart significantly in activity from a reference product, such that the current benefit-risk profile or effectiveness of the reference product will be significantly altered (when the p-biosim is used in other or all approved indications). Otherwise, it undermines the expected inter- and intra-lot variability in quality and other attributes that are typical and expected for the commercially available reference product and the p-biosim. Moreover, it is worth noting that the quality variability range for the reference product must have been used as boundary for where the p-biosim must operate, to be considered as such.

In conclusion, current trends in therapeutics increasingly demand the use of biologics. Access to biologics is a global challenge that could be partly ameliorated by the availability of biosimilars. The development of biosimilars is in its infancy. Despite global uniformity in the regulatory guidance pertaining to the clinical development of biosimilars, the emerging literature characterizing clinical development of p-biosims and arguments for extrapolation is not aligned with such guidances. There is an apparent gap in knowledge, expectations of the development process, and unwillingness to engage in biosimilars development that is seemingly related to access to biologics and mischaracterization of the clinical trials. All stakeholders should have a vested interest in this endeavor. Ultimately, a faster global adoption of biosimilars may enhance the goal of achieving better treatment outcomes promised by biotherapeutics as the next frontier in fighting a plethora of diseases.

\section{Acknowledgment}

The views expressed by the author are not an official position of Pfizer.

\section{Disclosure}

The author reports no conflicts of interest in this work.

\section{References}

1. U.S. Department of Health and Human Services, Food and Drug Administration. Guidance for Industry Scientific Considerations in Demonstrating Biosimilarity to a Reference Product. Rockville, MD: FDA; 2012.

2. European Medicines Agency. Guideline on Similar Biological Medicinal Products (Revision). CHMP/437/04 Rev 1. London: European Medicines Agency; 2014.

3. World Health Organization Expert Committee on Biological Standardization. Guidelines on Evaluation of Similar Biotherapeutic Products (SBP). Geneva, Switzerland: WHO; 2009.
Biosimilars

\section{Publish your work in this journal}

Biosimilars is an international, peer-reviewed, open access journal focusing on the manufacture, development and medicinal use of biopharmaceutical compounds considered similar to an innovator agent. Specific topics covered in the journal include: Regulatory issues and pathways; manufacturing processes; chemical composition and

\section{Dovepress}

structure; quality and purity; patent issues; bioequivalence and interchangeability; clinical efficacy data; patient perspectives. The manuscript management system is completely online and includes a very quick and fair peer-review system. Visit http://www.dovepress.com/ testimonials.php to read real quotes from published authors. 